\title{
Growth of Sobolev norms for abstract linear Schrödinger equations
}

\author{
D. Bambusi*, B. Grébert ${ }^{\dagger}$, A. Maspero ${ }^{\ddagger}$ D. Robert ${ }^{\S}$
}

November 6, 2018

\begin{abstract}
We prove an abstract theorem giving a $\langle t\rangle^{\epsilon}$ bound $(\forall \epsilon>0)$ on the growth of the Sobolev norms in linear Schrödinger equations of the form i $\dot{\psi}=H_{0} \psi+V(t) \psi$ when the time $t \rightarrow \infty$. The abstract theorem is applied to several cases, including the cases where (i) $H_{0}$ is the Laplace operator on a Zoll manifold and $V(t)$ a pseudodifferential operator of order smaller than 2; (ii) $H_{0}$ is the (resonant or nonresonant) Harmonic oscillator in $\mathbb{R}^{d}$ and $V(t)$ a pseudodifferential operator of order smaller than $H_{0}$ depending in a quasiperiodic way on time. The proof is obtained by first conjugating the system to some normal form in which the perturbation is a smoothing operator and then applying the results of [MR17].
\end{abstract}

\section{Introduction}

In this paper we study growth of Sobolev norms for solutions of the abstract linear Schrödinger equation

$$
\mathrm{i} \partial_{t} \psi=H_{0} \psi+V(t) \psi
$$

in a scale of Hilbert spaces $\mathcal{H}^{r}$; here $V(t)$ is a time dependent operator and $H_{0}$ a time independent linear operator. We will prove some abstract results

\footnotetext{
*Dipartimento di Matematica Federigo Enriques, Università degli Studi di Milano, Via Saldini 50, I-20133 Milano, Italy

Email: dario.bambusi@unimi.it

${ }^{\dagger}$ Laboratoire de Mathématiques Jean Leray, Université de Nantes, 2 rue de la Houssinière BP 92208, 44322 Nantes Cedex 3, France

Email: benoit.grebert@univ-nantes.fr

${ }^{\ddagger}$ International School for Advanced Studies (SISSA), Via Bonomea 265, 34136, Trieste, Italy

Email: alberto.maspero@sissa.it

${ }^{\S}$ Laboratoire de Mathématiques Jean Leray, Université de Nantes, 2 rue de la Houssinière BP 92208, 44322 Nantes Cedex 3, France

Email: didier.robert@univ-nantes.fr
} 
ensuring that for any $r \geq 0$ and any $\epsilon>0$, the $\mathcal{H}^{r}$ norm of the solution grows in time at most as $\langle t\rangle^{\epsilon}$ as $t \rightarrow \infty$, where $\langle t\rangle:=\sqrt{1+t^{2}}$. The main novelty of our results is that they allow (1) to weaken the standard gap assumptions on the spectrum of $H_{0}$, in particular to deal with some cases where the gaps are dense in $\mathbb{R}$, and (2) to deal with perturbations which are of any order strictly smaller than that of $H_{0}$ (see below for a precise definition).

The main applications are to the case where

(i) $H_{0}$ is either the Laplace operator on a Zoll manifold (e.g. the spheres) or an anharmonic oscillator in $\mathbb{R}$, while $V$ is an operator depending arbitrarily on time and having order strictly smaller than $H_{0}$;

(ii) $H_{0}$ is the (possibly nonresonant) multidimensional Harmonic oscillator and $V(t)$ is an operator which depends on time in a quasiperiodic way and has order strictly smaller than $H_{0}$.

Further applications will be presented in the paper.

We emphasize in particular the results (ii) which, as far as we know are the first controlling growth of Sobolev norms in higher dimensional systems without any gap condition.

The proof is based on the combination of the ideas of Bam17a, Bam17b, BGMR17] (which in turn are a developments of the ideas of [BBM14, see also [PT01, IPT05]) and the results of [MR17]; precisely, for any positive $N$, we construct a (finite) sequence of unitary time dependent transformations conjugating $H_{0}+V(t)$ to a Hamiltonian of the form

$$
H_{0}+Z^{(N)}(t)+V^{(N)}(t),
$$

where $\left[H_{0} ; Z^{(N)}\right]=0$ and $V^{(N)}$ is a smoothing operator of order $N$, namely an operator belonging to $\mathcal{L}\left(\mathcal{H}^{s} ; \mathcal{H}^{s+N}\right)$ for any $s$ (linear bounded operators from $\mathcal{H}^{s}$ to $\left.\mathcal{H}^{s+N}\right)$. Then we apply Theorem 1.5 of [MR17] to (1.2) getting the $\langle t\rangle^{\epsilon}$ bound on the growth of Sobolev norms.

We think that a further point of interest of our paper is that the conjugation to a system of the form (1.2) is here developed in an abstract context, instead then in the framework of classes of pseudodifferential operators adapted to the situation under study; this is the main reason why we get an abstract theory directly applicable to many different contexts.

The main point is that we introduce an abstract graded algebra of operators whose properties mimic the properties of pseudodifferential operators. The use of this framework is made possible by the technique we develop to solve the homological equations met in the construction of the conjugation of $H$ to (1.2). Indeed, we recall that in previous papers the smoothing theorem, namely the result conjugating the original system to (1.2) was obtained by quantizing the procedure of classical normal form. Here instead, we work directly at the quantum level, in particular solving at this level the two homological equations that we find (see eqs. (3.17) and (3.24) below). 
It is worth to add a few words on the way we solve the homological equations. When dealing with systems related to the applications (i), we assume that $H_{0}=f\left(K_{0}\right)$ where $f$ is a superlinear function and $K_{0}$ is an operator s.t.

$$
\operatorname{spec}\left(K_{0}\right) \subset \mathbb{N}+\lambda, \quad \lambda>0 .
$$

In this case we solve the homological equation essentially by averaging over the flow $e^{-\mathrm{i} t K_{0}}$ of $K_{0}$. In turn this is made possible by the use of a commutator expansion lemma proved in [DG97]. When dealing with the $d$ dimensional harmonic oscillators instead, we take

$$
H_{0}=\sum_{j=0}^{d} \nu_{j} K_{j},
$$

with $K_{j}$ commuting linear operators, each one fulfilling the property (1.3) (think of $K_{j}=-\partial_{x_{j}}^{2}+x_{j}^{2}$ ) and $\nu_{j}>0$; then we consider operators of the form

$$
\mathrm{e}^{\mathrm{i} \tau \cdot K} A \mathrm{e}^{-\mathrm{i} \tau \cdot K}
$$

(where of course $\tau \cdot K:=\tau_{1} K_{1}+\ldots+\tau_{d} K_{d}$ ), remark that they are quasiperiodic in the "angles" $\tau$, and use a Fourier expansion in $\tau$ in order to solve the homological equation.

The study of growth of Sobolev norms and the related results on the nature of the spectrum of the Floquet operator has a long history: we recall the results by How89, How92, Joy94 showing that the Floquet spectrum of systems with growing gaps and bounded perturbations is pure point, a result which implies boundedness of the expectation value of the energy. The first $\langle t\rangle^{\epsilon}$ estimates on the expectation value of the energy for system of the form (1.1) was obtained by Nenciu in Nen97 for the case of increasing gaps and bounded perturbations (see also BJ98, Joy96 for similar results), and by Duclos, Lev and Sťovíček [DLS08] in case of shrinking gaps. In the case of increasing gaps, such results were improved recently by two of us (see [MR17]) who obtained the $\langle t\rangle^{\epsilon}$ growth of Sobolev norms also in the case of unbounded perturbations depending arbitrarily on time, for example in the case where $H_{0}=-\partial_{x}^{2}+x^{2 k}$, the result of [MR17] allows to deal with perturbations growing at infinity as $|x|^{m}$ with $m<k-1$. In the present paper we get the result for any $m<2 k$. The result of [MR17 also applies to perturbations of the free Schrödiger equation on Zoll manifolds with perturbations of order strictly smaller than 1 . Here we deal with perturbations of order strictly smaller than 2. A study of perturbations of maximal order has been done independently by Montalto [Mon17] who got a control of the growth of Sobolev norms for the Schrödinger equation on $\mathbb{T}$ with $H=a(t, x)\left|-\partial_{x x}\right|^{M}+V(t)$ with $M>1 / 2$, $a$ a smooth positive function and $V$ a pseudodifferential operator of order smaller than $M$. 
Finally we recall that in MR17] logarithmic estimates for the growth of Sobolev norms were also obtained in the case of perturbations depending analytically on time. Here we do not attack the problem of getting logarithmic estimates, but we think that our technique would also allow to get such estimates.

A remarkable further result was obtained by Bourgain [Bou99] who obtained a logarithmic bound on the growth of Sobolev norms for the Schrödinger equation on $\mathbb{T}^{d}(d=1,2)$ in the case of an analytic perturbation depending quasiperiodically on time. Such a result is based on the use of a Lemma on the clustering of resonant sites (in a suitable space time lattice) which does not seem to extend to different geometries. The result of Bourgain was extended by Wang Wan08 to deal with Schrödinger equations on $\mathbb{T}$ perturbed by a potential analytic in time (but otherwise depending arbitrary on time) and greatly simplified by Delort Del10 who used it in an abstract framework which allows to deal with the case of $\mathbb{T}^{d}$ (any $d \geq 1$ ) and also with the case of Zoll manifolds, obtaining a growth bounded by $\langle t\rangle^{\epsilon}$ (see also FZ12 for analytic potentials on $\mathbb{T}^{d}$ ). We also mention the reducibility result by [EK09] dealing with small quasiperiodic perturbations of the free Schrödinger equation on $\mathbb{T}^{d}$; for such a system, the authors prove that growth of Sobolev norms cannot happen, provided the frequency of the quasiperiodic solution is chosen in a nonresonant set. At present our method does not allow to deal with the Schrödinger equation on $\mathbb{T}^{d}$ for $d \geq 2$.

Concerning Harmonic oscillators in $\mathbb{R}^{d}$ with $d>1$, a couple of reducibility results are known, namely GP16 in which the authors study small bounded perturbations of the completely resonant Harmonic oscillator, and [BGMR17] in which we studied small polynomial perturbations of the resonant or nonresonant Harmonic oscillator.

As far as we know no results are known on growth of Sobolev norms for perturbations of the harmonic oscillator:

$$
H_{0}:=-\Delta+\sum_{j=1}^{d} \nu_{j}^{2} x_{j}^{2},
$$

with nonresonant frequencies $\nu_{j}$. This is due to the fact that the differences between two of its eigenvalues $\left\{\lambda_{a}\right\}_{a \in \mathbb{N}^{d}}$, namely

$$
\lambda_{a}-\lambda_{b}=\nu \cdot(a-b)
$$

are dense on the real axis and this prevents the use of any previous technique. As anticipated above here we obtain the $\langle t\rangle^{\epsilon}$ growth for the case of perturbation of order strictly smaller than the order of the Harmonic oscillator. 
Acknowledgments. During the preparation of this work, we were supported by ANR -15-CE40-0001-02 "BEKAM" of the Agence Nationale de la Recherche. A. Maspero is also partially supported by PRIN 2015 "Variational methods, with applications to problems in mathematical physics and geometry".

\section{Main results}

\subsection{An abstract graded algebra}

We start with a Hilbert space $\mathcal{H}$ and a reference operator $K_{0}$, which we assume to be selfadjoint and positive, namely such that

$$
\left\langle\psi ; K_{0} \psi\right\rangle \geq c_{K}\|\psi\|^{2}, \quad \forall \psi \in D\left(K_{0}^{1 / 2}\right), \quad c_{K}>0,
$$

and define as usual a scale of Hilbert spaces by $\mathcal{H}^{r}=D\left(K_{0}^{r}\right)$ (the domain of the operator $K_{0}^{r}$ ) if $r \geq 0$, and $\mathcal{H}^{r}=\left(\mathcal{H}^{-r}\right)^{\prime}$ (the dual space) if $r<0$. Finally we denote by $\mathcal{H}^{-\infty}=\bigcup_{r \in \mathbb{R}} \mathcal{H}^{r}$ and $\mathcal{H}^{+\infty}=\bigcap_{r \in \mathbb{R}} \mathcal{H}^{r}$. We endow $\mathcal{H}^{r}$ with the natural norm $\|\psi\|_{r}:=\left\|\left(K_{0}\right)^{r} \psi\right\|_{0}$, where $\|\cdot\|_{0}$ is the norm of $\mathcal{H}^{0} \equiv \mathcal{H}$. Notice that for any $m \in \mathbb{R}, \mathcal{H}^{+\infty}$ is a dense linear subspace of $\mathcal{H}^{m}$ (this is a consequence of the spectral decomposition of $K_{0}$ ).

We introduce now a graded algebra $\mathcal{A}$ of operators which mimic some fundamental properties of different classes of pseudo-differential operators. For $m \in \mathbb{R}$ let $\mathcal{A}_{m}$ be a linear subspace of $\bigcap_{s \in \mathbb{R}} \mathcal{L}\left(\mathcal{H}^{s}, \mathcal{H}^{s-m}\right)$ and define $\mathcal{A}:=\bigcup_{m \in \mathbb{R}} \mathcal{A}_{m}$. We notice that the space $\bigcap_{s \in \mathbb{R}} \mathcal{L}\left(\mathcal{H}^{s}, \mathcal{H}^{s-m}\right)$ is a Fréchet space equipped with the semi-norms: $\|A\|_{m, s}:=\|A\|_{\mathcal{L}\left(\mathcal{H}^{s}, \mathcal{H}^{s-m}\right)}$.

One of our aims is to control the smoothing properties of the operators in the scale $\left\{\mathcal{H}^{r}\right\}_{r \in \mathbb{R}}$. If $A \in \mathcal{A}_{m}$ then $A$ is more and more smoothing if $m \rightarrow-\infty$ and the opposite as $m \rightarrow+\infty$. We will say that $A$ is of order $m$ if $A \in \mathcal{A}_{m}$.

Definition 2.1. We say that $S \in \mathcal{L}\left(\mathcal{H}^{+\infty}, \mathcal{H}^{-\infty}\right)$ is $N$-smoothing if $\forall \kappa \in \mathbb{R}$, it can be extended to an operator in $\mathcal{L}\left(\mathcal{H}^{\kappa}, \mathcal{H}^{\kappa+N}\right)$. When this is true for every $N \geq 0$, we say that $S$ is a smoothing operator.

The first set of assumptions concerns the properties of $\mathcal{A}_{m}$ :

\section{Assumption I:}

(i) For each $m \in \mathbb{R}, K_{0}^{m} \in \mathcal{A}_{m}$; in particular $K_{0}$ is an operator of order one.

(ii) For each $m \in \mathbb{R}, \mathcal{A}_{m}$ is a Fréchet space for a family of semi-norms $\left\{\wp_{j}^{m}\right\}_{j \geq 1}$ such that the embedding $\mathcal{A}_{m} \hookrightarrow \bigcap_{s \in \mathbb{R}} \mathcal{L}\left(\mathcal{H}^{s}, \mathcal{H}^{s-m}\right)$ is continuous.

If $m^{\prime} \leq m$ then $\mathcal{A}_{m^{\prime}} \subseteq \mathcal{A}_{m}$ with a continuous embedding. 
(iii) $\mathcal{A}$ is a graded algebra, i.e $\forall m, n \in \mathbb{R}$ : if $A \in \mathcal{A}_{m}$ and $B \in \mathcal{A}_{n}$ then $A B \in \mathcal{A}_{m+n}$ and the map $(A, B) \mapsto A B$ is continuous from $\mathcal{A}_{m} \times \mathcal{A}_{n}$ into $\mathcal{A}_{m+n}$.

(iv) $\mathcal{A}$ is a graded Lie-algebra 1 : if $A \in \mathcal{A}_{m}$ and $B \in \mathcal{A}_{n}$ then the commutator $[A, B] \in \mathcal{A}_{m+n-1}$ and the map $(A, B) \mapsto[A, B]$ is continuous from $\mathcal{A}_{m} \times \mathcal{A}_{n}$ into $\mathcal{A}_{m+n-1}$.

(v) $\mathcal{A}$ is closed under perturbation by smoothing operators in the following sense: let $A$ be a linear map: $\mathcal{H}^{+\infty} \rightarrow \mathcal{H}^{-\infty}$. If there exists $m \in \mathbb{R}$ such that for every $N>0$ we have a decomposition $A=A^{(N)}+S^{(N)}$, with $A^{(N)} \in \mathcal{A}_{m}$ and $S^{(N)}$ is $N$-smoothing, then $A \in \mathcal{A}_{m}$.

(vi) If $A \in \mathcal{A}_{m}$ then also the adjoint operator $A^{*} \in \mathcal{A}_{m}$. The duality here is defined by the scalar product $\langle\cdot, \cdot\rangle$ of $\mathcal{H}=\mathcal{H}^{0}$. The adjoint $A^{*}$ is defined by $\langle u, A v\rangle=\left\langle A^{*} u, v\right\rangle$ for $u, v \in \mathcal{H}^{\infty}$ and extended by continuity.

It is well known that classes of pseudo-differential operators satisfy these properties, provided one chooses for $K_{0}$ a suitable operator of the right order (see e.g. Hör85).

In Gui85] V. Guillemin has introduced abstract pseudo-differential algebras, called generalized Weyl algebras. For his purpose Gui85] needs different properties than ours, but obviously there is an overlap with our presentation.

Remark 2.2. One has that $\forall A \in \mathcal{A}_{m}, \forall B \in \mathcal{A}_{n}$

$$
\begin{aligned}
& \forall m, s \exists N \text { s.t. }\|A\|_{m, s} \leq C_{1} \wp_{N}^{m}(A), \\
& \forall m, n, j \quad \exists N \text { s.t. } \wp_{j}^{m+n}(A B) \leq C_{2} \wp_{N}^{m}(A) \wp_{N}^{n}(B), \\
& \forall m, n, j \quad \exists N \text { s.t. } \wp_{j}^{m+n-1}([A, B]) \leq C_{3} \wp_{N}^{m}(A) \wp_{N}^{n}(B),
\end{aligned}
$$

for some positive constants $C_{1}(s, m), C_{2}(m, n, j), C_{3}(m, n, j)$.

For $\Omega \subset \mathbb{R}^{d}$ and $\mathcal{F}$ a Fréchet space, we will denote by $C_{b}^{m}(\Omega, \mathcal{F})$ the space of $C^{m}$ maps $f: \Omega \ni x \mapsto f(x) \in \mathcal{F}$, such that, for every seminorm $\|\cdot\|_{j}$ of $\mathcal{F}$ one has

$$
\sup _{x \in \Omega}\left\|\partial_{x}^{\alpha} f(x)\right\|_{j}<+\infty, \quad \forall \alpha \in \mathbb{N}^{d}:|\alpha| \leq m .
$$

If (2.4) is true $\forall m$, we say $f \in C_{b}^{\infty}(\Omega, \mathcal{F})$.

The next property needed is the following Egorov property, also well known for pseudo-differential operators.

\footnotetext{
${ }^{1}$ This property will impose the choice of the semi-norms $\left\{\wp_{j}^{m}\right\}_{j \geq 1}$. We will see in the examples that the natural choice $\left(\|\cdot\|_{m, s}\right)_{s \geq 0}$ has to be refined.
} 
Assumption II: For any $A \in \mathcal{A}_{m}$ and $\tau \in \mathbb{R}$, the map $\tau \mapsto A(\tau):=$ $\mathrm{e}^{\mathrm{i} \tau K_{0}} A \mathrm{e}^{-\mathrm{i} \tau K_{0}} \in C_{b}^{0}\left(\mathbb{R}, \mathcal{A}_{m}\right)$.

Remark 2.3. From Assumption II one has that, for any $B \in \mathcal{A}_{n}$, for any $\ell \in \mathbb{N}, \operatorname{ad}_{A(s)}^{\ell}(B) \in C_{b}^{0}(]-T, T\left[, \mathcal{A}_{n+(m-1) \ell}\right), \forall T>0$. Here $\operatorname{ad}_{A}(B):=$ $\mathrm{i}[A, B]$.

Remark that Assumption II is a quantum property for the time evolution of observables. Practically it follows from the time evolution of classical observables (Hamilton equation) if some classes of symbols are preserved under the classical flows. Indeed one might replace Assumption II by a weaker one (see Appendix B).

\subsection{Perturbations of systems of order larger than 1}

Now we state our spectral assumption on $K_{0}$ :

Assumption A : $K_{0}$ has an entire discrete spectrum such that

$$
\operatorname{spec}\left(K_{0}\right) \subseteq \mathbb{N}+\lambda
$$

for some $\lambda>0$.

Our second spectral assumption is essentially that the unperturbed operator $H_{0}$ is a function of $K_{0}$. To state it precisely we need the following definition

Definition 2.4. A function $f \in C^{\infty}(\mathbb{R})$ will be said to be a classical symbol of order $\rho(a t+\infty)$ if there exist real numbers $\left\{c_{j}\right\}_{j \geq 0}$ s.t. $c_{0} \geq 0$ and for all $k \geq 1$, all $N \geq 1$, there exists $C_{k, N}$ s.t.

$$
\left|\frac{d^{k}}{d x^{k}}\left(f(x)-\sum_{0 \leq j \leq N-1} c_{j} x^{\rho-j}\right)\right| \leq C_{k, N}\left|x^{\rho-N-k}\right|, \quad \forall x \geq 1 .
$$

We will denote by $S^{\rho}$ the space of classical symbols of order $\rho$.

We shall say that $f$ is an elliptic classical symbol of order $\rho$ if $f$ is real and $c_{0}>0$. We shall write $f \in S_{+}^{\rho}$.

We shall say that $f$ is a classical symbol of order $-\infty$ if $f \in S^{m} \forall m<0$. We shall write $f \in S^{-\infty}$.

Some standard properties of classical symbols are recalled in Appendix A. We assume that

Assumption B: There exists an elliptic classical symbol $f$ of order $\mu>1$, such that

$$
H_{0}=f\left(K_{0}\right)
$$


We will prove (see Lemma A.2) that (2.6) implies $H_{0} \in \mathcal{A}_{\mu}$, i.e. $H_{0}$ is an operator of order $\mu>1$.

We come back to the Schrödinger equation defined by the time dependent Hamiltonian $H(t):=H_{0}+V(t)$ (see (1.10). When the solution $\psi(t)$ exists globally in time, we define the Schrödinger propagator $\mathcal{U}(t, s)$, generated by (1.1), such that

$$
\psi(t)=\mathcal{U}(t, s) \psi, \quad \mathcal{U}(s, s)=\mathbf{1}
$$

We are ready to state our main result on systems with increasing gaps:

Theorem 2.5. Assume that $\mathcal{A}$ is a graded algebra as defined in Section 2.1] and that $K_{0}, H_{0}$ satisfy assumptions $A$ and $B$. Furthermore assume that the perturbation $V(t)$ with domain $\mathcal{H}^{\infty}$ is symmetric for every $t \in \mathbb{R}$ and satisfies

$$
V \in C_{b}^{\infty}\left(\mathbb{R}, \mathcal{A}_{\rho}\right), \quad \text { with } \rho<\mu .
$$

Then $H(t)=H_{0}+V(t)$ generates a propagator $\mathcal{U}(t, s)$ s.t. $\mathcal{U}(t, s) \in \mathcal{L}\left(\mathcal{H}^{r}\right)$ $\forall r \in \mathbb{R}$.

Moreover for any $r>0$ and any $\epsilon>0$ there exists $C_{r, \epsilon}>0$ such that

$$
\|\mathcal{U}(t, s) \psi\|_{r} \leq C_{r, \epsilon}\langle t-s\rangle^{\epsilon}\|\psi\|_{r}, \quad \forall t, s \in \mathbb{R} .
$$

This result extends a result by Nenciu [Nen97] for bounded perturbations $(\rho=0)$. Furthermore in [MR17] two of us had already extended Nenciu's result to unbounded perturbations with the constraint $\rho<\min (\mu-1,1)$. The main point is that we add here a stronger spectral assumption: essentially the spectrum of $H_{0}$ is $f(\mathbb{N}+\lambda)$ for some smooth function $f$ (see Assumptions $\mathrm{A}$ and $\mathrm{B})$.

As a final remark, we note that Theorem 2.5 gives also a proof of the existence and of some properties of the propagator $\mathcal{U}(t, s)$, which in the framework of Theorem 2.5 are not obvious.

\subsection{Applications (i)}

Zoll manifolds. Recall that a Zoll manifold is a compact Riemannian manifold $(M, g)$ such that all the geodesic curves have the same period $T:=2 \pi$. For example the $d$-dimensional sphere $\mathbb{S}^{d}$ is a Zoll manifold. We denote by $\triangle_{g}$ the positive Laplace-Beltrami operator on $M$ and by $H^{r}(M)=\operatorname{Dom}\left(1+\triangle_{g}\right)^{r / 2}, r \geq 0$, the usual scale of Sobolev spaces. Finally we denote by $S_{\mathrm{cl}}^{m}(M)$ the space of classical real valued symbols of order $m \in \mathbb{R}$ on the cotangent $T^{*}(M)$ of $M$ (see Hörmander Hör85] for more details).

Definition 2.6. We say that $A \in \mathcal{A}_{m}$ if it is a pseudodifferential operator (in the sense of Hörmander [Hör85]) with symbol of class $S_{\mathrm{cl}}^{m}(M)$. 
In this case the operator $K_{0}$ is a perturbation of order -1 of $\sqrt{\triangle_{g}}$ (see Sect. 4.1), and the norms $\|\psi\|_{r}$ coincide with the standard Sobolev norms.

Corollary 2.7 (Zoll manifolds). Let $V(t)$ be a symmetric pseudo-differential operator of order $\rho<2$ on $M$ such that its symbol $v \in C_{b}^{\infty}\left(\mathbb{R} ; S_{\mathrm{cl}}^{\rho}(M)\right)$. Then the propagator $\mathcal{U}(t, s)$ generated by $H(t)=\triangle_{g}+V(t)$ exists and satisfies (2.9).

Anharmonic oscillators on $\mathbb{R}$. The second application concerns one dimensional quantum anharmonic oscillators

$$
\mathrm{i} \partial_{t} \psi=H_{k, l} \psi+V(t) \psi, \quad x \in \mathbb{R},
$$

where $H_{k, l}$ is the one degree of freedom Hamiltonian

$$
H_{k, l}:=D_{x}^{2 l}+a x^{2 k}, \quad k, l \in \mathbb{N}, \quad k+l \geq 3, \quad a>0 .
$$

Here $D_{x}:=\mathrm{i}^{-1} \partial_{x}$. It is well known that $H_{k, \ell}$ is essentially self-adjoint in $L^{2}(\mathbb{R})$ HR82b.

Define the Sobolev spaces $\mathcal{H}^{r}:=\operatorname{Dom}\left(H_{k, l}^{\frac{k+l}{2 k l} r}\right)$ for $r \geq 0$. We define now suitable operator classes for the perturbation. Denote

$$
\mathrm{k}_{0}(x, \xi):=\left(1+x^{2 k}+\xi^{2 l}\right)^{\frac{k+l}{2 k l}} .
$$

Definition 2.8. A function $f$ will be called a symbol of order $\rho \in \mathbb{R}$ if $f \in C^{\infty}\left(\mathbb{R}_{x} \times \mathbb{R}_{\xi}\right)$ and $\forall \alpha, \beta \in \mathbb{N}$, there exists $C_{\alpha, \beta}>0$ s.t.

$$
\left|\partial_{x}^{\alpha} \partial_{\xi}^{\beta} f(x, \xi)\right| \leq C_{\alpha, \beta} \mathrm{k}_{0}(x, \xi)^{\rho-\frac{k \beta+l \alpha}{k+l}} .
$$

We will write $f \in S_{\text {an }}^{\rho}$.

As usual to a symbol $f \in S_{\text {an }}^{\rho}$ we associate the operator $f\left(x, D_{x}\right)$ which is obtained by standard Weyl quantization (see formula (4.2) below).

Definition 2.9. We say that $F \in \mathcal{A}_{\rho}$ if it is a pseudodifferential operator with symbol of class $S_{\mathrm{an}}^{\rho}$, i.e., if there exist $f \in S_{\mathrm{an}}^{\rho}$ and $S$ smoothing (in the sense of Definition [2.1) such that $F=f\left(x, D_{x}\right)+S$.

In this case the seminorms are defined by

$$
\wp_{j}^{\rho}(F):=\sum_{|\alpha|+|\beta| \leq j} C_{\alpha \beta}
$$

with $C_{\alpha \beta}$ the smallest constants s.t. eq. (2.12) holds. If a symbol $f$ depends on additional parameters (e.g. it is time dependent), we ask that the constants $C_{\alpha, \beta}$ are uniform w.r.t. such parameters. 
Remark 2.10. With this definition of symbols, one has $x \in S_{\mathrm{an}}^{\frac{l}{k+l}}, \xi \in S_{\mathrm{an}}^{\frac{k}{k+l}}$, $x^{2 k}+\xi^{2 l} \in S_{\mathrm{an}}^{\frac{2 k l}{k+l}}, \mathrm{k}_{0}(x, \xi) \in S_{\mathrm{an}}^{1}$.

We get the following:

Corollary 2.11 (1-D anharmonic oscillators). Consider equation (2.10) with the assumption (2.11). Assume also that $V \in C_{b}^{\infty}\left(\mathbb{R} ; \mathcal{A}_{\rho}\right)$ with $\rho<\frac{2 k l}{k+l}$. Then the propagator $\mathcal{U}(t, s)$ generated by $H(t)=H_{k, l}+V(t)$ is well defined and satisfies (2.9).

An example of admissible perturbation is $V(t, x, \xi)=\sum_{l \alpha+k \beta<2 k l} a_{\alpha, \beta}(t) x^{\alpha} \xi^{\beta}$

with $a_{\alpha, \beta} \in C_{b}^{\infty}(\mathbb{R}, \mathbb{R})$. In particular if we choose $H_{0}=-\frac{d^{2}}{d x^{2}}+x^{4}$, we can consider unbounded perturbations of the form $x^{3} g(t)$ and of course also $x g(t)$ with $g \in C_{b}^{\infty}(\mathbb{R}, \mathbb{R})$.

Remark 2.12. Our class of perturbations contains quite general pseudodifferential operators, however it is easy to see that multiplication operators (i.e. operators independent of $\partial_{x}$ ) must be polynomials in $x$ with coefficients which are possibly time dependent.

In the similar problem of reducibility more general classes of perturbations have been treated in Bam17b]. We did not try to push the result in that direction. This is probably non trivial in an abstract framework like the one we are using here.

Remark 2.13. We think that our method should also allow to deal with some perturbations of the same order as the main term. For example it should be treatable the case where $V$ is a quasihomogeneous polynomial of maximal order fulfilling some sign condition (more or less as in Theorem 2.12 of (Bam17a]).

\subsection{Perturbations of systems of order 1}

In order to deal with perturbations of operators of order 1 we have to restrict to the case where the dependence of the perturbation on time is quasiperiodic.

Let $\mathcal{A}:=\cup_{m \in \mathbb{R}} \mathcal{A}_{m}$ be a graded Lie algebra satisfying Assumption I with a reference operator $K_{0}$.

Let $K_{1}, K_{2}, \cdots, K_{d}$ be $d$ self-adjoint positive operators such that $K_{j} \in \mathcal{A}_{1}$, $\forall 1 \leq j \leq d$. Assume the following modified Assumption II:

\section{Assumption II':}

(i) $\left[K_{j}, K_{\ell}\right]=0$ for any $0 \leq j, \ell \leq d$. 
(ii) Denote $K=\left(K_{1}, \cdots, K_{d}\right)$ and for $\tau \in \mathbb{R}^{d}, \tau \cdot K:=\sum_{1 \leq j \leq d} \tau_{j} K_{j}$.

Then for any $A \in \mathcal{A}_{m}$, the map $\tau \mapsto A(\tau):=\mathrm{e}^{\mathrm{i} \tau \cdot K} A \mathrm{e}^{-\mathrm{i} \tau \cdot \bar{K}} \in C_{b}^{\infty}\left(\mathbb{R}^{d} ; \mathcal{A}_{m}\right)$.

Remark 2.14. For any $B \in \mathcal{A}_{n}$, for any $\ell \in \mathbb{N}$, one has $\operatorname{ad}_{A(s)}^{\ell}(B) \in$ $C_{b}^{\infty}\left(\mathbb{R}^{d} ; \mathcal{A}_{n+\ell(m-1)}\right)$.

We also adapt our spectral conditions:

Assumption $\mathbf{A}^{\prime}: K=\left(K_{1}, \cdots, K_{d}\right)$ has an entire joint spectrum, $\operatorname{spec}(K) \subseteq$ $\mathbb{N}^{d}+\lambda$ for some $\lambda \in \mathbb{R}^{d}, \lambda \geq 0$.

Assumption $\mathbf{B}^{\prime}$ : There exist $\left\{\nu_{j}\right\}_{j=1}^{d}, \nu_{j}>0$ s.t.

$$
\begin{array}{r}
H_{0}=\sum_{1 \leq j \leq d} \nu_{j} K_{j}, \\
K_{0}=H_{0} .
\end{array}
$$

In order to fix ideas one can think of the case of Harmonic oscillators, in which $K_{j}=-\partial_{j}^{2}+x_{j}^{2}, 1 \leq j \leq d$.

Remark 2.15. Since the operators $K_{j}$ are positive, the norm $\|\cdot\|_{r}$ defined using the operator $K_{0}$ is equivalent to the norm defined using the operator $K_{0}^{\prime}:=\sum_{j=1}^{d} K_{j}$.

We consider both the case where

$$
\nu:=\left(\nu_{1}, \ldots, \nu_{d}\right)
$$

is resonant and the case where it is nonresonant. To state the arithmetical assumptions on $\nu$, we first recall the following well known lemma whose scheme of proof will be recalled in the Appendix C

Lemma 2.16. There exists $\tilde{d} \leq d$, a vector $\tilde{\nu} \in \mathbb{R}^{\tilde{d}}$ with components independent over the rationals, and vectors $\mathbf{v}_{j} \in \mathbb{Z}^{d}, j=1, \ldots, \tilde{d}$ such that

$$
\nu=\sum_{j=1}^{\tilde{d}} \tilde{\nu}_{j} \mathbf{v}_{j} .
$$

Remark 2.17. For example

(i) if $\nu$ is nonresonant, then $\tilde{\nu}=\nu$ and $\mathbf{v}_{j}=\mathbf{e}_{j}$, the standard basis of $\mathbb{R}^{d}$;

(ii) if $\nu$ is completely resonant then $\tilde{d}=1$; e.g. if $\nu=(1, \ldots, 1)$, then $\tilde{\nu}_{1}=1$, $\mathbf{v}_{1}=(1, \ldots, 1)$. 
Theorem 2.18. Assume that $V(t)=W(\omega t)$ with $W \in C_{b}^{\infty}\left(\mathbb{T}^{n}, \mathcal{A}_{\rho}\right)$ a quasiperiodic operator of order $\rho<1$. Assume furthermore that $(\tilde{\nu}, \omega) \in \mathbb{R}^{\tilde{d}+n}$ is a Diophantine vector, namely that there exist $\gamma>0$, and $\kappa \in \mathbb{R}$ s.t.,

$$
|\omega \cdot k+\tilde{\nu} \cdot \ell| \geq \frac{\gamma}{(|\ell|+|k|)^{\kappa}}, \quad 0 \neq(k, \ell) \in \mathbb{Z}^{n+\tilde{d}} .
$$

Then the propagator $\mathcal{U}(t, s)$ generated by $H(t)=\nu \cdot K+W(\omega t)$ exists and satisfies (2.9).

Remark 2.19. The vector $\tilde{\nu}$ is defined up to linear combinations with integer coefficients; clearly condition (2.16) does not depend on the choice of $\tilde{\nu}$.

Remark 2.20. We recall that Diophantine vectors form a subset of $\mathbb{R}^{n+\tilde{d}}$ of full measure if $\kappa>n+\tilde{d}-1$.

\subsection{Applications (ii)}

Relativistic Schrödinger equation on Zoll manifolds. We consider the reduced Dirac equation on a Zoll manifold $M$ with mass $\mu>0$

$$
\mathrm{i} \partial_{t} \psi=\sqrt{\triangle_{g}+\mu} \psi+V\left(\omega t, x, D_{x}\right) \psi, \quad t \in \mathbb{R}, x \in M .
$$

As in the case of the Schrödinger equation on Zoll manifolds, $\mathcal{A}_{\rho}$ is the class of pseudodifferential operators with symbols in $S_{\mathrm{cl}}^{\rho}(M)$ (see Definition 2.6).

In this case $V$ is assumed to be quasi-periodic in time.

Corollary 2.21 (Relativistic Schrödinger equation on Zoll manifolds). Assume that $V(t)=W(\omega t)$ with $W \in C^{\infty}\left(\mathbb{T}^{n}, \mathcal{A}_{\rho}\right)$ with $\rho<1$. Assume furthermore that the non resonance condition

$$
|\omega \cdot k+m| \geq \frac{\gamma}{1+|k|^{\kappa}}, \quad \forall 0 \neq k \in \mathbb{Z}^{n}, \quad \forall m \in \mathbb{Z}
$$

holds for some $\gamma>0$ and $\kappa$. Then the propagator $\mathcal{U}(t, s)$ generated by $H(t)=\sqrt{\triangle_{g}+\mu}+W(\omega t)$ exists and satisfies (2.9).

Harmonic oscillator in $\mathbb{R}^{d}$. Consider the quantum Harmonic oscillator

$$
\begin{array}{r}
\mathrm{i} \partial_{t} \psi=H_{\nu} \psi+V(t) \psi, \quad x \in \mathbb{R}^{d} \\
H_{\nu}:=-\Delta+\sum_{j=1}^{d} \nu_{j}^{2} x_{j}^{2}, \quad V(t)=W\left(\omega t, x, D_{x}\right) .
\end{array}
$$

Here $W$ is the Weyl quantization of a symbol belonging to the following class 
Definition 2.22. A function $f$ will be called a symbol of order $\rho \in \mathbb{R}$ if $f \in C^{\infty}\left(\mathbb{R}_{x}^{d} \times \mathbb{R}_{\xi}^{d}\right)$ and $\forall \alpha, \beta \in \mathbb{N}^{d}$, there exists $C_{\alpha, \beta}>0$ s.t.

$$
\left|\partial_{x}^{\alpha} \partial_{\xi}^{\beta} f(x, \xi)\right| \leq C_{\alpha, \beta}\left(1+|x|^{2}+|\xi|^{2}\right)^{\rho-\frac{|\beta|+|\alpha|}{2}} .
$$

We will write $f \in S_{\text {ho }}^{\rho}$.

The class (2.20) is the extension to higher dimensions of the class used in the anharmonic oscillators (see Definition 2.8) and with $k=l=1$.

Remark 2.23. With our numerology, the symbol of the harmonic oscillator is of order $1,|\xi|^{2}+\sum_{j} \nu_{j}^{2} x_{j}^{2} \in S_{\text {ho }}^{1}$, and not of order 2 as typically in the literature.

The classes $\mathcal{A}_{m}$ are defined as in Definition 2.9, with symbols in the class $S_{\mathrm{ho}}^{m}$.

Corollary 2.24. Assume that $\nu$ is such that $\tilde{\nu}$ fulfills (2.16), and that $W \in$ $C^{\infty}\left(\mathbb{T}^{n} ; \mathcal{A}_{\rho}\right)$ with $\rho<1$. Then the propagator $\mathcal{U}(t, s)$ of $H(t)=H_{\nu}+W(\omega t)$ exists and fulfills (2.9).

Remark that after a trivial rescaling of the spatial variables, $H_{\nu}=$ $\sum_{j=1}^{d} \nu_{j}\left(-\partial_{j}^{2}+x_{j}^{2}\right)$, thus the corollary is a trivial application of Theorem 2.18

Remark 2.25. In the completely resonant case

$$
H_{(1, \ldots, 1)}=-\Delta+|x|^{2},
$$

one has $\tilde{\nu}=1$ and the set of the $\omega^{\prime}$ s for which (2.16) is fulfilled has full measure provided $\kappa>n$.

Remark 2.26. We note that in the resonant case there have been exhibited examples of polynomial growths of the Sobolev norms. In particular see [Del14] and [BGMR17] for periodic in time perturbations; of course in such examples the frequency $\omega$ does not fulfill (2.16). Finally we recall also [BJLPN], where some some random in time perturbations are considered.

\section{Proofs of the abstract theorems}

\subsection{Scheme of the proof}

As explained in the introduction, the main step of the proof consists in proving a theorem conjugating the original Hamiltonian to a Hamiltonian of the form (1.2); this will be done in Theorem 3.8. Subsequently we will apply Theorem 1.5 of [MR17, which essentially states that, if $H(t)$ is such that for some $N>-1$

$$
\left[H(t), K_{0}\right] K_{0}^{N} \in C_{b}^{0}\left(\mathbb{R}, \mathcal{L}\left(\mathcal{H}^{r}\right)\right),
$$


then $\exists C_{r, N}>0$ such that

$$
\|\mathcal{U}(t, s) \psi\|_{r} \leq C_{r, N}\langle t-s\rangle^{\frac{r}{1+N}}\|\psi\|_{r}, \quad \forall t, s \in \mathbb{R} .
$$

We come to the algorithm of conjugation of the original Hamiltonian to (1.2). Before discussing it, we need to know the way a Hamiltonian is changed by a time dependent unitary transformation. This is the content of the following lemma.

Lemma 3.1. Let $H(t)$ be a time dependent self-adjoint operator, and $X(t)$ be a selfadjoint family of operators. Assume that $\psi(t)=\mathrm{e}^{-\mathrm{i} X(t)} \varphi(t)$ then

$$
\mathrm{i} \dot{\psi}=H(t) \psi \quad \Longleftrightarrow \quad \mathrm{i} \dot{\varphi}=\tilde{H}(t) \varphi
$$

where

$$
\tilde{H}(t):=\mathrm{e}^{\mathrm{i} X(t)} H(t) \mathrm{e}^{-\mathrm{i} X(t)}-\int_{0}^{1} \mathrm{e}^{\mathrm{i} s X(t)} \dot{X}(t) \mathrm{e}^{-\mathrm{i} s X(t)} \mathrm{d} s .
$$

This is seen by an explicit computation. For example see Lemma 3.2 of Bam17a.

A further important property giving the expansion of an operator of the form $\mathrm{e}^{\mathrm{i} X(t)} A \mathrm{e}^{-\mathrm{i} X(t)}$ in operators of decreasing order is stated in the following lemma.

Lemma 3.2. Let $X \in \mathcal{A}_{\rho}$ with $\rho<1$ be a symmetric operator. Let $A \in \mathcal{A}_{m}$ with $m \in \mathbb{R}$. Then $X$ is selfadjoint and for any $M \geq 1$ we have

$$
\mathrm{e}^{\mathrm{i} \tau X} A \mathrm{e}^{-\mathrm{i} \tau X}=\sum_{\ell=0}^{M} \frac{\tau^{\ell}}{\ell !} \operatorname{ad}_{X}^{\ell}(A)+R_{M}(\tau, X, A), \quad \forall \tau \in \mathbb{R},
$$

where $R_{M}(\tau, X, A) \in \mathcal{A}_{m-(M+1)(1-\rho)}$.

In particular $\operatorname{ad}_{X}^{\ell}(A) \in \mathcal{A}_{m-\ell(1-\rho)}$ and $\mathrm{e}^{\mathrm{i} \tau X} A \mathrm{e}^{-\mathrm{i} \tau X} \in \mathcal{A}_{m}, \forall \tau \in \mathbb{R}$.

The proof will be given in Sect. 3.2 .

We describe now the algorithm which will lead to the smoothing Theorem 3.8 the proof is slightly different according to the set of assumptions one chooses. We start by discussing it under the assumptions of Theorem 2.5. namely Assumption A and B. Subsequently we will discuss the changes needed to deal with Theorem 2.18 ,

We look for a change of variables of the form $\varphi=\mathrm{e}^{\mathrm{i} X_{1}(t)} \psi$ where $X_{1}(t) \in$ $\mathcal{A}_{\rho-\mu+1}$ is a self-adjoint operator which, due to the assumption $\rho<\mu$, has order smaller then one. Then $\varphi$ fulfills the Schrödinger equation i $\dot{\varphi}=$ $H^{+}(t) \varphi$ with

$$
\begin{aligned}
H^{+}(t):= & \mathrm{e}^{\mathrm{i} X_{1}(t)} H(t) \mathrm{e}^{-\mathrm{i} X_{1}(t)}-\int_{0}^{1} \mathrm{e}^{\mathrm{i} s X_{1}(t)} \dot{X}_{1}(t) \mathrm{e}^{-\mathrm{i} s X_{1}(t)} \mathrm{d} s \\
= & H_{0}+\mathrm{i}\left[X_{1}(t), H_{0}\right]+V(t)+\mathrm{i}\left[X_{1}(t), V(t)\right]-\frac{1}{2}\left[X_{1}(t),\left[X_{1}(t), H_{0}\right]\right]+\cdots \\
& \quad-\int_{0}^{1} \mathrm{e}^{\mathrm{i} s X_{1}(t)} \dot{X}_{1}(t) \mathrm{e}^{-\mathrm{i} s X_{1}(t)} \mathrm{d} s .
\end{aligned}
$$


In view of the properties of the graded algebra we have $\left[X_{1}, V\right] \in \mathcal{A}_{2 \rho-\mu}$, $\left[X_{1},\left[X_{1}, H_{0}\right]\right] \in \mathcal{A}_{2 \rho-\mu}$ (Assumption I (iv)) and $\mathrm{e}^{\mathrm{i} s X_{1}(t)} \dot{X}_{1}(t) \mathrm{e}^{-\mathrm{i} s X_{1}(t)} \in$ $\mathcal{A}_{\rho-\mu+1}$ (Lemma 3.2), therefore one has

$$
H^{+}(t)=H_{0}+\mathrm{i}\left[X_{1}(t), H_{0}\right]+V(t)+V_{1}^{+}(t),
$$

with $V_{1}^{+}(t) \in C_{b}^{\infty}\left(\mathbb{R}, \mathcal{A}_{\min (\rho-\mu+1,2 \rho-\mu)}\right)$.

Now we look for $X_{1}(t)$ s.t.

$$
\mathrm{i}\left[H_{0}, X_{1}(t)\right]=V(t)-\langle V(t)\rangle,
$$

where $\langle V(t)\rangle$ is the average over $\tau$ of $e^{\mathrm{i} \tau K_{0}} V(t) e^{-\mathrm{i} \tau K_{0}}$ (see (3.18) ), which in particular commutes with $K_{0}$. We will verify in Lemma 3.5 that there exists $X_{1}$ s.t.

$$
\mathrm{i}\left[H_{0}, X_{1}(t)\right]-V(t)+\langle V(t)\rangle \in \mathcal{A}_{\rho-1} .
$$

Therefore using such a $X_{1}$ to generate a unitary transformation, we get

$$
H^{+}(t):=H_{0}+\langle V(t)\rangle+V^{+}(t)
$$

where $V^{+}(t) \in C_{b}^{\infty}\left(\mathbb{R}, \mathcal{A}_{\rho-\delta}\right)$ with

$$
\delta:=\min (1, \mu-1, \mu-\rho)>0 .
$$

Therefore $V^{+}(t)$ is a perturbation of order lower than $V(t)$. Furthermore $\langle V(t)\rangle$ commutes with $K_{0}$.

Iterating this procedure we will establish an "almost" reducibility result that will be stated and proved in Subsect. 3.4.

Then, using Theorem 1.5 of [MR17, we immediately get Theorem 2.5.

In the case where $H_{0} \in \mathcal{A}_{1}$ the procedure has to be slightly modified since in this case $X_{1}$ and therefore $\dot{X}_{1}$ has the same order as $V$ and thus it cannot be considered as a remainder when analyzing $H^{+}$. In this case one rewrites

$$
\begin{aligned}
H^{+}(t) & =H_{0}+\mathrm{i}\left[X_{1}(t), H_{0}\right]+V(t) \\
& +\mathrm{i}\left[X_{1}(t), V(t)\right]-\frac{1}{2}\left[X_{1}(t),\left[X_{1}(t), H_{0}\right]\right]+\cdots \\
& -\dot{X}_{1}-\int_{0}^{1}\left(\mathrm{i} s\left[X_{1}(t), \dot{X}_{1}(t)\right]+\ldots .\right) \mathrm{d} s,
\end{aligned}
$$

so that eq. (3.6) is substituted by

$$
H^{+}(t)=H_{0}+\mathrm{i}\left[X(t), H_{0}\right]+V(t)-\dot{X}_{1}(t)+V^{+}(t),
$$

with $V^{+} \in \mathcal{A}_{\rho-\delta_{*}}$,

$$
\delta_{*}:=1-\rho>0,
$$


so again it is more regular than $V(t)$. Thus one is led to consider the new homological equation

$$
\mathrm{i}\left[H_{0}, X_{1}(t)\right]+\dot{X}_{1}(t)=V(t)-\langle V(t)\rangle,
$$

where $\langle V(t)\rangle$ has to commute with $K_{0}$. In order to be able to solve such an equation we restrict to the case of $V(t)$ quasiperiodic in $t$ and, as explained in the introduction, we develop a procedure based on a suitable Fourier expansion to construct $X_{1}$ and $\langle V(t)\rangle$. The details are given in Lemma 3.7 which will ensure that such a homological equation has a smooth solution and thus the procedure is well defined also in the case of order 1.

\subsection{A couple of lemmas on flows}

Lemma 3.3. (i) Let $X \in \mathcal{A}_{1}$ be symmetric w.r.t. the scalar product of $\mathcal{H}^{0}$. Then $X$ has a unique self-adjoint extension and $\mathrm{e}^{-\mathrm{i} \tau X} \in \mathcal{L}\left(\mathcal{H}^{r}\right) \forall r \geq 0$ and $\forall \tau \in \mathbb{R}$. Furthermore $\mathrm{e}^{-\mathrm{i} \tau X}$ is an isometry in $\mathcal{H}^{0}$.

(ii) Assume that $X(t)$ is a family of symmetric operators in $\mathcal{A}_{1}$ s.t.

$$
\sup _{t \in \mathbb{R}} \wp_{j}^{1}(X(t))<\infty, \quad \forall j \geq 1 .
$$

Then there exist $c_{r}, C_{r}>0$ s.t.

$$
c_{r}\|\psi\|_{r} \leq\left\|\mathrm{e}^{-\mathrm{i} \tau X(t)} \psi\right\|_{r} \leq C_{r}\|\psi\|_{r}, \quad \forall t \in \mathbb{R}, \quad \forall \tau \in[0,1] .
$$

Proof. (i) From the properties of the algebra $\mathcal{A}$ we have that $X K_{0}^{-1}$ and $\left[X, K_{0}\right] K_{0}^{-1}$ are of order 0 . Thus by definition these operators belong to $\mathcal{L}\left(\mathcal{H}^{r}\right) \forall r \in \mathbb{R}$. Then the result follows from Theorem 1.2 of [MR17.

(ii) By item (i), for any $t \in \mathbb{R}$ and $\tau \in[0,1]$ the operator $\mathrm{e}^{-\mathrm{i} \tau X(t)}$ is an isometry in $\mathcal{H}^{0}$, therefore

$$
\left\|\mathrm{e}^{-\mathrm{i} \tau X(t)} \psi\right\|_{r}=\left\|\mathrm{e}^{\mathrm{i} \tau X(t)} K_{0}^{r} \mathrm{e}^{-\mathrm{i} \tau X(t)} \psi\right\|_{0} .
$$

Then we have

$$
\begin{gathered}
\mathrm{e}^{\mathrm{i} \tau X(t)} K_{0}^{r} \mathrm{e}^{-\mathrm{i} \tau X(t)} \psi=K_{0}^{r} \psi+\mathrm{i} \int_{0}^{\tau} \mathrm{e}^{\mathrm{i} \tau_{1} X(t)}\left[X(t), K_{0}^{r}\right] \mathrm{e}^{-\mathrm{i} \tau_{1} X(t)} \psi \mathrm{d} \tau_{1} \\
=K_{0}^{r} \psi+\mathrm{i} \int_{0}^{\tau} \mathrm{e}^{\mathrm{i} \tau_{1} X(t)}\left[X(t), K_{0}^{r}\right] K_{0}^{-r} K_{0}^{r} \mathrm{e}^{-\mathrm{i} \tau_{1} X(t)} \psi \mathrm{d} \tau_{1}
\end{gathered}
$$

By the properties of the algebra $\mathcal{A}$ and (3.13) one has that (using (2.1) $-(2.3)$ )

$$
\sup _{t \in \mathbb{R}}\left\|\left[X(t), K_{0}^{r}\right] K_{0}^{-r}\right\|_{\mathcal{L}\left(\mathcal{H}^{0}\right)}<C_{r}<+\infty
$$

therefore taking the norm $\|\cdot\|_{0}$ of (3.15) one gets the inequality

$$
\left\|\mathrm{e}^{-\mathrm{i} \tau X(t)} \psi\right\|_{r} \leq\|\psi\|_{r}+\int_{0}^{\tau} C_{r}\left\|\mathrm{e}^{-\mathrm{i} \tau_{1} X(t)} \psi\right\|_{r} \mathrm{~d} \tau_{1}
$$


Then by Gronwall we conclude that

$$
\left\|\mathrm{e}^{-\mathrm{i} \tau X(t)} \psi\right\|_{r} \leq \mathrm{e}^{C_{r}}\|\psi\|_{r}, \quad \forall t \in \mathbb{R}, \quad \forall \tau \in[-1,1] .
$$

This proves the majoration in (3.14). The minoration follows simply by the identity $\psi=e^{\mathrm{i} \tau X(t)} e^{-\mathrm{i} \tau X(t)} \psi$ and the majoration.

Proof of Lemma 3.2. Selfadjointness was proven in the previous lemma. Let us apply to the 1.h.s. of (3.5) the Taylor formula at $\tau=0$. Then we get, with $U_{X}(\tau):=\mathrm{e}^{-\mathrm{i} \tau X}$ and $\operatorname{ad}_{X}(A):=\mathrm{i}[X, A]$

$$
\begin{aligned}
& U_{X}(-\tau) A U_{X}(\tau) \\
& =\sum_{j=0}^{M} \frac{\tau^{j}}{j !} \operatorname{ad}_{X}^{j}(A)+\frac{\tau^{M+1}}{M !} \int_{0}^{1}(1-s)^{M+1} U_{X}(-s \tau) \operatorname{ad}_{X}^{M+1}(A) U_{X}(s \tau) \mathrm{d} s
\end{aligned}
$$

Using Assumption I (iv), we have $\operatorname{ad}_{X}^{j}(A) \in \mathcal{A}_{m-j(1-\rho)}$. We define the remainder $R_{M}(\tau, X, A)$ to be the integral term in (3.16), which, using also Lemma 3.3, belongs to $\mathcal{L}\left(\mathcal{H}^{s}, \mathcal{H}^{s-m+(M+1)(1-\rho)}\right), \forall s \in \mathbb{R}$. Therefore the remainder $R_{M}(\tau, X, A)$ is $N$-smoothing provided $M+1 \geq \frac{N+m}{1-\rho}$. As $M$ can be taken arbitrary large, $\mathrm{e}^{\mathrm{i} \tau X} A \mathrm{e}^{-\mathrm{i} \tau X}$ fulfills Assumption I (v), thus it belongs to $\mathcal{A}_{m}$.

\subsection{Solution of the Homological equations}

The first homological equation. As we have seen in Section 3.1, to prove Theorem 2.5 we need to study an homological equation of the form

$$
\mathrm{i}\left[H_{0}, X\right]=A-\langle A\rangle,
$$

where $A \in \mathcal{A}_{m}$ and $\langle A\rangle$ is the average of $A$ along the periodic flow of $K_{0}$ :

$$
\langle A\rangle:=\frac{1}{2 \pi} \int_{0}^{2 \pi} A(\tau) \mathrm{d} \tau, \quad A(\tau)=\mathrm{e}^{\mathrm{i} \tau K_{0}} A \mathrm{e}^{-\mathrm{i} \tau K_{0}} .
$$

Notice that the assumption on the spectrum of $K_{0}$ (see Assumption A) entails that $\mathrm{e}^{2 \mathrm{i} \pi K_{0}}=\mathrm{e}^{2 \mathrm{i} \pi \lambda}$, thus for any $A \in \mathcal{A}$ one has $\mathrm{e}^{2 \mathrm{i} \pi K_{0}} A \mathrm{e}^{-2 \mathrm{i} \pi K_{0}}=A$, namely $\tau \mapsto A(\tau)$ is $2 \pi$ periodic.

Lemma 3.4. Let $A \in \mathcal{A}_{m}, m \in \mathbb{R}$. Then $\langle A\rangle \in \mathcal{A}_{m}$ and

$$
\left[K_{0},\langle A\rangle\right]=0 \text {. }
$$

Proof. $\langle A\rangle \in \mathcal{A}_{m}$ is a consequence of Assumption II. Identity (3.19) follows by a direct computation. 
Lemma 3.5. (i) Let $A \in \mathcal{A}_{m}, m \in \mathbb{R}$. Then

$$
Y=\frac{1}{2 \pi} \int_{0}^{2 \pi} \tau(A-\langle A\rangle)(\tau) \mathrm{d} \tau
$$

solves the homological equation

$$
\mathrm{i}\left[K_{0}, Y\right]=A-\langle A\rangle .
$$

Further $Y \in \mathcal{A}_{m}$ and if $A$ is symmetric, so is $Y$.

(ii) Choose $R>0$ such that $f^{\prime}(x) \geq 1$ if $x \geq R$ and $\eta \in C^{\infty}(\mathbb{R})$ such that $\eta(x)=1$ if $x \in[0, R], \eta(x)=0$ if $x \geq R+1$. Define

$$
X:=\left(1-\eta\left(K_{0}\right)\right)\left(f^{\prime}\left(K_{0}\right)\right)^{-1} Y,
$$

with $Y$ as in (3.20). Then $X \in \mathcal{A}_{m-\mu+1}$, is symmetric provided $A$ is symmetric and solves (3.17) modulo an error term in $\mathcal{A}_{m-1}$. More precisely

$$
\mathrm{i}\left[H_{0}, X\right]=A-\langle A\rangle+\mathcal{A}_{m-1} .
$$

We note for the sequel that if $A \in \mathcal{A}_{m}$ then $X \in \mathcal{A}_{m-(\mu-1)}$, namely we have a gain of $\mu-1>0$ in the smoothing order.

Proof. Assertion (i) is proved by integration by parts using that $A(\tau)$ is $2 \pi$ periodic.

To prove (ii), first remark that by Assumption B and Lemma A.1, $f^{\prime} \in S_{+}^{\mu-1}$, thus it is different from zero provided $x \geq R$ is large enough. It follows that the function $x \mapsto \frac{1-\eta(x)}{f^{\prime}(x)} \in S^{-\mu+1}$. Therefore, by Lemma A.2, the operator $\left(1-\eta\left(K_{0}\right)\right)\left(f^{\prime}\left(K_{0}\right)\right)^{-1} \in \mathcal{A}_{-\mu+1}$. Finally since $Y \in \mathcal{A}_{m}$, it follows that $X \in \mathcal{A}_{m-\mu+1}$.

We show now that $X$ solves (3.23). This is a consequence of the commutator expansion Lemma. Indeed fix $N \geq 2$, then by Lemma A.3 one has

$$
\left[H_{0}, X\right]=\left[f\left(K_{0}\right), X\right]=f^{\prime}\left(K_{0}\right)\left[K_{0}, X\right]+\sum_{2 \leq j \leq N} \frac{1}{j !} f^{(j)}\left(K_{0}\right) \operatorname{ad}_{K_{0}}^{j}(X)+R_{N+1}(f, X)
$$

with $R_{N+1}(f, X) \in \mathcal{A}_{m-\mu+1+[\mu]-N} \subset \mathcal{A}_{m-1}$.

By Lemma A.1 and Assumption I, for any integer $j \geq 2$ one has that $f^{(j)}\left(K_{0}\right) \operatorname{ad}_{K_{0}}^{j}(X) \in \mathcal{A}_{m-\mu+1+\mu-j} \subset \mathcal{A}_{m-1}$. Then we get

$$
\begin{aligned}
\mathrm{i}\left[H_{0}, X\right] & =\mathrm{i} f^{\prime}\left(K_{0}\right)\left[K_{0}, X\right]+A_{m-1} \\
& \stackrel{\sqrt{3.22}}{=}\left(1-\eta\left(K_{0}\right)\right) \mathrm{i}\left[K_{0}, Y\right]+A_{m-1} \\
& \stackrel{(3.21)}{=}\left(1-\eta\left(K_{0}\right)\right)(A-\langle A\rangle)+A_{m-1},
\end{aligned}
$$

with $A_{m-1} \in \mathcal{A}_{m-1}$. Now put $R:=-\eta\left(K_{0}\right)(A-\langle A\rangle)$. Since $x \mapsto \eta(x) \in$ $S^{-\infty}, R$ is a smoothing operator and thus $A_{m-1}+R \in \mathcal{A}_{m-1}$. 
The second homological equation. We want to solve eq. (3.12). Using the quasiperiodicity assumption $V(t)=W(\omega t)$, we look for a quasiperiodic solution $X_{1}(t)=X(\omega t)$ of the equation

$$
\omega \cdot \partial_{\theta} X(\omega t)+\mathrm{i}\left[H_{0}, X(\omega t)\right]=W(\omega t)-\langle W(\omega t)\rangle .
$$

In order to define precisely $\langle W(\omega t)\rangle$, consider again the vectors $\mathbf{v}_{j}$ and the frequencies $\tilde{\nu}_{j}$ of Lemma 2.16. First remark that, since $\nu=\sum_{j=1}^{\tilde{d}} \tilde{\nu}_{j} \mathbf{v}_{j}$, one has $\nu \cdot K=\sum_{j=1}^{\tilde{d}}\left(K \cdot \mathbf{v}_{j}\right) \tilde{\nu}_{j}$, so that, defining

$$
\tilde{K}_{j}:=K \cdot \mathbf{v}_{j}, \quad \tilde{K}:=\left(\tilde{K}_{1}, \ldots, \tilde{K}_{\tilde{d}}\right),
$$

one has

$$
H_{0} \equiv \nu \cdot K=\tilde{\nu} \cdot \tilde{K},
$$

and furthermore, since $\mathbf{v}_{j}$ has integer entries, then the joint spectrum of $\tilde{K} \equiv\left(\tilde{K}_{1}, \ldots, \tilde{K}_{\tilde{d}}\right)$ is s.t. $\operatorname{spec}(\tilde{K}) \subset \mathbb{Z}^{\tilde{d}}+\tilde{\lambda}$, therefore the map $\mathbb{R}^{\tilde{d}} \ni \tau \mapsto$ $A(\tau):=e^{\mathrm{i} \tau \cdot \tilde{K}} A e^{-\mathrm{i} \tau \cdot \tilde{K}}$ is periodic in each of the $\tau_{j}{ }^{\prime}$ 's. Define now

$$
\langle A\rangle:=\frac{1}{(2 \pi)^{\tilde{d}}} \int_{\mathbb{T}^{\tilde{d}}} \mathrm{e}^{\mathrm{i} \tau \cdot \tilde{K}} A \mathrm{e}^{-\mathrm{i} \tau \cdot \tilde{K}} \mathrm{~d} \tau .
$$

Remark 3.6. Let $A \in \mathcal{A}_{m}, m \in \mathbb{R}$. Then by Assumption $I I^{\prime},\langle A\rangle \in \mathcal{A}_{m}$ and

$$
\left[\tilde{K}_{j},\langle A\rangle\right]=0, \quad 1 \leq j \leq \tilde{d} ; \quad\left[K_{0},\langle A\rangle\right]=0 .
$$

Lemma 3.7. Let $A \in C_{b}^{\infty}\left(\mathbb{T}^{n}, \mathcal{A}_{m}\right), m \in \mathbb{R}$. Provided (2.16) holds, the homological equation (3.24) has a solution $X \in C^{\infty}\left(\mathbb{T}^{n}, \mathcal{A}_{m}\right)$. Furthermore if $A$ is symmetric then $X$ is symmetric as well.

Proof. For $A \in C^{\infty}\left(\mathbb{T}^{n}, \mathcal{A}_{m}\right)$, denote $A^{\sharp}(\theta, \tau):=\mathrm{e}^{\mathrm{i} \tau \cdot \tilde{K}} A(\theta) \mathrm{e}^{-\mathrm{i} \tau \cdot \tilde{K}}$. By Assumption $\mathrm{II}^{\prime}, A^{\sharp} \in C^{\infty}\left(\mathbb{T}^{n+\tilde{d}}, \mathcal{A}_{m}\right)$. Since $A^{\sharp}$ is defined on $\mathbb{T}^{n+\tilde{d}}$, we can expand it in Fourier series:

$$
A^{\sharp}(\theta, \tau)=\sum_{(k, \ell) \in \mathbb{Z}^{n+\tilde{d}}} \hat{A}_{k, \ell}^{\sharp} \mathrm{e}^{\mathrm{i}(k \cdot \theta+\ell \cdot \tau)},
$$

where

$$
\hat{A}_{k, \ell}^{\sharp}:=\frac{1}{(2 \pi)^{n+\tilde{d}}} \int_{\mathbb{T}^{n+\tilde{d}}} A^{\sharp}(\theta, \tau) \mathrm{e}^{-\mathrm{i}(k \cdot \theta+\ell \cdot \tau)} \mathrm{d} \theta \mathrm{d} \tau .
$$

Notice that

$$
A(\theta) \equiv A^{\sharp}(\theta, 0)=\sum_{(k, \ell) \in \mathbb{Z}^{n+\tilde{d}}} \hat{A}_{k, \ell^{\sharp}}^{\mathrm{i} k \cdot \theta} .
$$

Then, instead of solving directly the homological equation (3.24), we solve $\omega \cdot \partial_{\theta} X^{\sharp}(\theta, \tau)+\mathrm{i}\left[H_{0}, X^{\sharp}(\theta, \tau)\right]=(W-\langle W\rangle)^{\sharp}(\theta, \tau), \quad \forall \theta \in \mathbb{T}^{n}, \quad \forall \tau \in \mathbb{T}^{\tilde{d}}$. 
Clearly if we find a smooth solution $X^{\sharp}(\theta, \tau)$ of this equation, then $X(\theta):=$ $X^{\sharp}(\theta, 0)$ solves the original homological equation (3.24). Now remark that

$$
\begin{aligned}
\mathrm{i}\left[H_{0}, X^{\sharp}(\theta, \tau)\right] & =\sum_{j=1}^{\tilde{d}} \tilde{\nu}_{j} \mathrm{i}\left[\tilde{K}_{j}, X^{\sharp}(\theta, \tau)\right]=\left.\sum_{j=1}^{\tilde{d}} \tilde{\nu}_{j} \frac{d}{d \epsilon}\right|_{\epsilon=0} \mathrm{e}^{\mathrm{i} \epsilon \tilde{K}_{j}} X^{\sharp}(\theta, \tau) \mathrm{e}^{-\mathrm{i} \epsilon \tilde{K}_{j}} \\
& =\left.\sum_{j=1}^{\tilde{d}} \tilde{\nu}_{j} \frac{d}{d \epsilon}\right|_{\epsilon=0} X^{\sharp}\left(\theta, \tau+\epsilon \mathbf{e}_{j}\right) \\
& =\left.\sum_{(k, \ell) \in \mathbb{Z}^{n+\tilde{d}}} \hat{X}_{k, \ell}^{\sharp} \frac{d}{d \epsilon}\right|_{\epsilon=0} \sum_{j=1}^{\tilde{d}} \tilde{\nu}_{j} \mathrm{e}^{\mathrm{i}\left(k \cdot \theta+\ell \cdot\left(\tau+\epsilon \mathbf{e}_{j}\right)\right)} \\
& =\sum_{(k, \ell) \in \mathbb{Z}^{n+\tilde{d}}} \mathrm{i} \tilde{\nu} \cdot \ell \hat{X}_{k, \ell}^{\sharp} \mathrm{e}^{\mathrm{i}(k \cdot \theta+\ell \cdot \tau)} .
\end{aligned}
$$

Therefore, expanding in Fourier series, equation (3.29) is equivalent to

$$
\left.\mathrm{i}(\omega \cdot k+\tilde{\nu} \cdot \ell) \hat{X}_{k, \ell}^{\sharp}=(\widehat{W-\langle W}\rangle\right)_{k, \ell}^{\sharp} \cdot
$$

Hence define

$$
\hat{X}_{k, \ell}^{\sharp}=-\mathrm{i} \frac{(\widehat{W-\langle W})_{k, \ell}^{\sharp}}{(\omega \cdot k+\tilde{\nu} \cdot \ell)}, \quad \text { if } \omega \cdot k+\tilde{\nu} \cdot \ell \neq 0 .
$$

Since $W^{\sharp}$ is in $C^{\infty}\left(\mathbb{T}^{n+\tilde{d}}, \mathcal{A}_{m}\right)$ we get that for any $j, N \geq 1$ there exists $C_{N, j}$ such that

$$
\wp_{j}^{m}\left((\widehat{W-\langle W\rangle})_{k, \ell}^{\sharp}\right) \leq C_{N, j}(|k|+|\ell|)^{-N} .
$$

So we get easily that if $X$ is defined by $X(\theta)=X^{\sharp}(\theta, 0)$ and $X^{\sharp}$ has Fourier coefficients (3.30) with $X_{k, 0}^{\sharp}=0$, then $X \in C_{b}^{\infty}\left(\mathbb{T}^{n}, \mathcal{A}_{m}\right)$.

\subsection{The iterative Lemma}

We state and prove the iterative Lemma which is the main step for the proof of our main results.

Theorem 3.8. Assume that the assumptions of Theorem 2.5 or of Theorem 2.18 are satisfied.

There exist $\delta>0$ and a sequence $\left\{X_{j}(t)\right\}_{j \geq 1}$ of self-adjoint (time-dependent) operators in $\mathcal{H}$ with $X_{j} \in C_{b}^{\infty}\left(\mathbb{R}, \mathcal{A}_{\rho-(\mu-1)-(j-1) \delta}\right)$, such that $\forall j$, the inequalities (3.14) are satisfied; for any $N \geq 1$ the change of variables

$$
\psi=e^{-\mathrm{i} X_{1}(t)} \ldots e^{-\mathrm{i} X_{N}(t)} \varphi
$$


transforms $H_{0}+V(t)$ into the Hamiltonian

$$
H^{(N)}(t):=H_{0}+Z^{(N)}(t)+V^{(N)}(t)
$$

where $Z^{(N)} \in C_{b}^{\infty}\left(\mathbb{R}, \mathcal{A}_{\rho}\right)$ commutes with $K_{0}$, i.e. $\left[Z^{(N)}, K_{0}\right]=0$, while $V^{(N)} \in C_{b}^{\infty}\left(\mathbb{R}, \mathcal{A}_{\rho-N \delta}\right)$. Furthermore, under the assumptions of Theorem 2.18, one has

$$
\left[Z^{(N)} ; \tilde{K}_{j}\right]=0, \quad \forall j=1, \ldots, \tilde{d} .
$$

Proof. It is proved by recurrence. Consider first the assumptions of Theorem 2.5. Using Lemmas 3.1, 3.2, 3.3, 3.5, 3.7 one gets the theorem for $N=1$ with $Z^{(1)}(t):=\langle V(t)\rangle \in C_{b}^{\infty}\left(\mathbb{R}, \mathcal{A}_{\rho}\right)$. By Lemma 3.4. $\left[Z^{(1)}(t), K_{0}\right]=0$. In this case $\delta$ can be taken as in (3.9).

The iterative step $N \rightarrow N+1$ is proved following the same lines, just adding the remark that $e^{\mathrm{i} X_{N+1}} Z^{(N)} e^{-\mathrm{i} X_{N+1}}-Z^{(N)} \in \mathcal{A}_{\rho-(\mu-1)-N \delta+\rho-1} \subset$ $\mathcal{A}_{\rho-(N+1) \delta}$.

Under the assumptions of Theorem 2.18, the result is proved along the same lines, with $\delta$ as in (3.11). The property (3.33) follows by Remark 3.6 .

\subsection{Proof of Theorem 2.5}

By Theorem 3.8, the operator $H(t)$ is conjugated to $H^{(N)}(t)$. So we apply Theorem 1.5 of [MR17] to the Schrödinger equation for $H^{(N)}(t)$. More precisely we have

$$
\left[H^{(N)}(t), K_{0}\right]=\left[V^{(N)}(t), K_{0}\right] \in C_{b}^{0}\left(\mathbb{R}, \mathcal{A}_{\rho-N \delta}\right)
$$

and thus, by choosing $N$ large enough, (3.2) ensures the result for the propagator $\mathcal{U}_{N}(t, s)$ of $H^{(N)}(t)$.

Now since $H(t)$ is conjugated to $H^{(N)}(t), H(t)$ generates a propagator $\mathcal{U}(t, s)$ in the Hilbert space scale $\mathcal{H}^{r}$ unitarily equivalent to the propagator $\mathcal{U}_{N}(t, s)$. Therefore, using also (3.14), the propagator $\mathcal{U}(t, s)$ fulfills (2.9), thus yielding the result.

\section{Applications}

In this section we prove Corollary 2.7, Corollary 2.11 and Corollary 2.21.

\subsection{Zoll manifolds}

To begin with we show how to put ourselves in the abstract setup. So first we define the operator $K_{0}$. This will be achieved by exploiting the spectral properties of the operator $\triangle_{g}$. Applying Theorem 1 of Colin de Verdière [CdV79], there exists a pseudodifferential operator $Q$ of order -1 , 
commuting with $\triangle_{g}$, such that $\operatorname{Spec}\left[\sqrt{\triangle_{g}}+Q\right] \subseteq \mathbb{N}+\lambda$ with some $\lambda \geq 0$. We can assume $\lambda>0$. If not, denoting $\Pi_{-}$the projector on the non positive eigenvalues, we replace $Q$ by $Q+C \Pi_{-}$with $C>0$ large enough; remark that $\Pi_{-}$commutes with $\triangle_{g}$ and is a smoothing operator. So we define

$$
K_{0}:=\sqrt{\triangle_{g}}+Q, \quad H_{0}:=K_{0}^{2} .
$$

Now remark that $H_{0}=\triangle_{g}+2 Q \sqrt{\triangle_{g}}+Q^{2}$, so we have

$$
H_{0}=\triangle_{g}+Q_{0}
$$

where $Q_{0}$ is a pseudo-differential operator of order 0 and therefore

$$
H(t)=\triangle_{g}+V(t) \equiv H_{0}+\tilde{V}(t), \quad \tilde{V}(t):=V(t)-Q_{0}
$$

and we are in the setup of the abstract Schrödinger equation (1.1) with the new perturbation $\tilde{V}(t)$.

Remark that $\mathcal{H}^{r}:=\operatorname{Dom}\left(\left(K_{0}\right)^{r}\right), r \geq 0$, coincides with the classical Sobolev space $H^{r}(M)$ and one has the equivalence of norms

$$
c_{r}\|\psi\|_{H^{r}(M)} \leq\|\psi\|_{r} \leq C_{r}\|\psi\|_{H^{r}(M)}, \quad \forall r \in \mathbb{R} .
$$

We define the class $\mathcal{A}_{m}$ to be the class of pseudodifferential operators whose (real valued) symbols belong to $S_{\mathrm{cl}}^{m}(M)$. Clearly $K_{0} \in \mathcal{A}_{1}$ (recall that $\Pi_{-}$is a smoothing operator). It is classical that Assumptions I and II are fulfilled Hör85.

Proof of Corollary 2.7. Assumption A holds true by construction of $K_{0}$, Assumption B holds with $f(x)=x^{2}$ and therefore $\mu:=2$. Since $V(t)$ is a pseudodifferential operator of order $\rho<2$ whose symbol belongs to $C_{b}^{\infty}\left(\mathbb{R}, S_{\mathrm{cl}}^{\rho}(M)\right)$, one verifies easily, using pseudodifferential calculus (in particular estimates (2.1) -(2.3) $)$, that $\tilde{V}(t)=V(t)-Q_{0} \in C_{b}^{\infty}\left(\mathbb{R}, \mathcal{A}_{\rho}\right)$. Hence the corollary follows from Theorem 2.5 .

\subsection{Anharmonic oscillators}

We recall that for a symbol $a$ (in the sense of Definition 2.8) we denote by $a\left(x, D_{x}\right)$ its Weyl quantization

$$
\left(a\left(x, D_{x}\right) \psi\right)(x):=\frac{1}{2 \pi} \iint_{y, \xi \in \mathbb{R}} \mathrm{e}^{\mathrm{i}(x-y) \xi} a\left(\frac{x+y}{2}, \xi\right) \psi(y) \mathrm{d} y \mathrm{~d} \xi .
$$

We endow $S_{\text {an }}^{\rho}$ (defined in Definition 2.8) with the family of seminorms

$$
\wp_{j}^{\rho}(a):=\sum_{|\alpha|+|\beta| \leq j} \sup _{(x, \xi) \in \mathbb{R}^{2}} \frac{\left|\partial_{x}^{\alpha} \partial_{\xi}^{\beta} a(x, \xi)\right|}{\left[\mathrm{k}_{0}(x, \xi)\right]^{\rho-\frac{k \beta+l \alpha}{k+l}}}, \quad j \in \mathbb{N} .
$$


The operator $K_{0}$ is defined using the spectral properties of the Hamiltonian $H_{k, l}$ defined in (2.11) that were studied in detail in [HR82b]; in that paper an accurate Bohr-Sommerfeld rule for the the eigenvalues of $H_{k \downarrow l}$ was obtained and the existence of a pseudodifferential operator $Q$ of ordet $2-1$ such that $\operatorname{Spec}\left[H_{k, l}^{\frac{k+l}{2 k l}}+Q\right] \subseteq \mathbb{N}+\lambda(\lambda \geq 0)$ was proven. Note that for our numerology $H_{k, l}^{\frac{k+l}{2 k l}}$ is of order 1 by definition. Therefore we define

$$
K_{0}:=H_{k, l}^{\frac{k+l}{2 k l}}+Q, \quad H_{0}:=K_{0}^{\frac{2 k l}{k+l}} .
$$

We define $\mathcal{A}_{m}$ to be the class of pseudodifferential operator with symbols in $S_{\text {an }}^{m}$. Notice that by construction $\mathcal{A}_{m} \subset \mathcal{L}\left(\mathcal{H}^{s}, \mathcal{H}^{s-m}\right)$ for all $s \in \mathbb{R}$. It is classical that $\mathcal{A}$ fulfills Assumptions I and II (see [HR82b, HR82a).

On the other hand Assumptions A and B are fulfilled with $\mu:=\frac{2 k l}{k+l}>1$ (as $k+l \geq 3)$. Furthermore one has

$$
H_{k, \ell}=\left(K_{0}-Q\right)^{\frac{2 k l}{k+l}}=K_{0}^{\frac{2 k l}{k+l}}+Q_{0}
$$

where $Q_{0}$ is a pseudodifferential operator of order $\frac{2 k l}{k+l}-2$. Therefore

$$
H(t)=H_{k, l}+V(t) \equiv H_{0}+\tilde{V}(t), \quad \tilde{V}(t):=V(t)+Q_{0}
$$

and once again we are in the setup of the abstract Schrödinger equation (1.1) with the new perturbation $\tilde{V}(t)$.

Proof of Corollary 2.11. Since $V(t)$ is a pseudodifferential operator of order $\rho<\frac{2 k l}{k+l}$ whose symbol and its time-derivatives have uniformly (in time) bounded seminorms, one verifies that $\tilde{V}(t)=V(t)+Q_{0} \in C_{b}^{\infty}\left(\mathbb{R}, \mathcal{A}_{\rho}\right)$. Hence the corollary follows from Theorem 2.5 .

\subsection{Relativistic Schrödinger equation on Zoll manifolds}

The proof of Corollary 2.21 is along the lines developed in Subsection 4.1 , Let us remark that the operator $\sqrt{\triangle_{g}+\mu}-\sqrt{\triangle_{g}}$ is of order -1 . Hence, defining $K_{0}$ as in (4.1), one has again $\sqrt{\triangle_{g}+\mu}=K_{0}+Q_{0}$ with $Q_{0}$ of order -1 . Therefore

$$
H(t)=\sqrt{\triangle_{g}+\mu}+V\left(\omega t, x, D_{x}\right)=K_{0}+\tilde{V}(\omega t)
$$

\footnotetext{
${ }^{2}$ Actually [HR82b] proves that $Q$ has a symbol which is quasi-homogeneous of degree $-k-l$. Here a symbol $f(x, \xi)$ is quasi-homogeneous of degree $m$ if

$$
f\left(\lambda^{l} x, \lambda^{k} \xi\right)=\lambda^{m} f(x, \xi), \quad \forall \lambda>0, \quad \forall(x, \xi) \in \mathbb{R}^{2} \backslash\{0\} .
$$

It is classical HR82b, HR82a that if $f$ is quasi-homogeneous of degree $m$, then it is a symbol in the class $S_{\text {an }}^{m /(k+l)}$.
} 
with the new perturbation $\tilde{V}(\omega t) \in C^{\infty}\left(\mathbb{T}^{n}, \mathcal{A}_{\rho}\right)$.

This time we verify Assumptions $\mathrm{II}^{\prime}, \mathrm{A}^{\prime}$ and $\mathrm{B}^{\prime}$ with $d=1$ and $K_{1}=$ $K_{0}=H_{0}$. Concerning the nonresonance condition just remark that in this case we have that $\nu$ has only one component given by 1 .

Thus Theorem 2.18 immediately yields Corollary 2.21

\section{A Technical lemmas on classical symbols}

We begin with the following lemma whose proof is completely standard (and we skip it)

Lemma A.1. (i) If $f \in S^{a}, g \in S^{b}$ then $f g \in S^{a+b}$.

(ii) If $f \in S^{a}$, then $f^{(j)} \in S^{a-j}$.

(iii) If $x \mapsto \eta(x)$ is a smooth cut-off function on $\mathbb{R}$, then $\eta \in S^{-\infty}$.

(iv) The function $f(x)=x^{a}, a>0$, is a classical elliptic symbol in $S_{+}^{a}$.

Lemma A.2. If $g \in S^{\mu}, \mu \in \mathbb{R}$, then $g\left(K_{0}\right) \in \mathcal{A}_{\mu}$.

Proof. By definition $g(x)=\sum_{0 \leq j \leq N-1} c_{j} x^{\mu-j}+R(x),|R(x)| \leq C_{N}\left|x^{\mu-N}\right|$ for $|x| \geq 1$. Then $g\left(K_{0}\right)=\sum_{0 \leq j \leq N-1} c_{j} K_{0}^{\mu-j}+R\left(K_{0}\right)$, where $R\left(K_{0}\right)$ is defined by functional calculus as $R\left(K_{0}\right):=\int_{0}^{\infty} R(\lambda) \mathrm{d} E_{K_{0}}(\lambda)$, $\mathrm{d} E_{K_{0}}(\lambda)$ being the spectral resolution of $K_{0}$. By Assumption I, $\sum_{0 \leq j \leq N-1} c_{j} K_{0}^{\mu-j} \in \mathcal{A}_{\mu}$ while the operator $R\left(K_{0}\right)$ is $N$-smoothing (in the sense of Definition 2.1). Since $N$ can be taken arbitrarily large, $g\left(K_{0}\right)$ fulfills Assumption I (v), therefore it belongs to $\mathcal{A}_{\mu}$. The other properties are easily verified using such decomposition.

Finally, we recall a commutator expansion lemma following from [DG97, Lemma C.3.1]:

Lemma A.3. Let $f \in S_{+}^{\rho}$ and $W \in \mathcal{A}_{m}$. Then for all $N \geq[\rho]$ we have

$$
\left[f\left(K_{0}\right), W\right]=\sum_{1 \leq j \leq N} \frac{1}{j !} f^{(j)}\left(K_{0}\right) \operatorname{ad}_{K_{0}}^{j} W+R_{N+1}\left(f, K_{0}, W\right),
$$

where $R_{N+1}\left(f, K_{0}, W\right) \in \mathcal{A}_{[\rho]+m-N}$.

Moreover if $W$ depends on time $t$ with uniform estimates in $\mathcal{A}_{m}$ then it is also true for $R_{N+1}\left(f, K_{0}, W\right)$.

Proof. Apply [DG97, Lemma C.3.1] to the bounded operator $B=K_{0}^{-m} W$. 


\section{B An abstract proof of Egorov Theorem}

In order to check Assumption II, we introduce the following condition

Assumption II-CL: For every $m \in \mathbb{R}$ and every $A \in \mathcal{A}_{m}$ there exists $\Phi^{(t)}(A) \in C_{b}^{1}\left(\mathbb{R}_{t}, \mathcal{A}_{m}\right)$ and $R(A, t) \in C_{b}^{0}\left(\mathbb{R}_{t}, \mathcal{A}_{m-1}\right)$ such that $\Phi^{(0)}(A)=A$ and

$$
\frac{d}{d t} \Phi^{(t)}(A)=\mathrm{i}^{-1}\left[\Phi^{(t)}(A), K_{0}\right]+R(A, t)
$$

In applications in a pseudodifferential operator setting, we have $A=O p(a)$, $a$ is the symbol of $A$ and one can choose $\Phi^{(t)}(A)=O p\left(a \circ \phi^{t}\right)$ where $\phi^{t}$ is the classical flow of the symbol of $K_{0}$. Then one has to verify that $a \circ \phi^{t}$ belongs to the same symbol class as $a$ (see for example Tay91]).

Theorem B.1 (Abstract Egorov Theorem). If Assumption I and Assumption II-CL are satisfied then Assumption II holds true.

Proof. We follow Rob87] (p. 202-207). Let $U(t)=\mathrm{e}^{-\mathrm{i} t K_{0}}$. Compute

$$
\begin{aligned}
& \frac{d}{d \tau}\left(U(\tau-t) \Phi^{(\tau)}(A) U(t-\tau)\right) \\
& \quad=U(\tau-t)\left(\mathrm{i}\left[\Phi^{(\tau)}(A), K_{0}\right]+\frac{d}{d \tau} \Phi^{(\tau)}(A)\right) U(t-\tau) .
\end{aligned}
$$

So using (B.1) and integrate in $\tau$ between 0 and $t$ we get

$$
U(-t) A U(t)=\Phi^{(t)}(A)+\int_{0}^{t} U(\tau-t) R(A, \tau) U(t-\tau) \mathrm{d} \tau .
$$

Now we iterate from this formula. In the following step we apply this formula for every $\tau$ to $A_{\text {new }}=R(A, \tau)$. So we get

$$
\begin{aligned}
U(-t) A U(t) & =A_{0}(t)+A_{1}(t) \\
+ & \int_{0}^{t} \int_{0}^{t-\tau} U\left(\tau+\tau_{1}-t\right) R\left(R(A, \tau), \tau-\tau_{1}\right) U\left(t-\tau-\tau_{1}\right) \mathrm{d} \tau \mathrm{d} \tau_{1} .
\end{aligned}
$$

where $A_{0}(t)=\Phi^{(t)}(A), A_{1}(t)=\int_{0}^{t} \Phi^{(t-\tau)}(R(A, \tau)) \mathrm{d} \tau \in \mathcal{A}_{m-1}$ and $R\left(R(A, \tau), \tau-\tau_{1}\right) \in \mathcal{A}_{m-2}$.

At the step $N$ we get easily by induction:

$$
\begin{aligned}
& U(-t) A U(t)=A_{0}(t)+A_{1}(t)+\cdots+A_{N}(t) \\
& \quad+\int_{0}^{t} \int_{0}^{t-\tau_{0}} \cdots \int_{0}^{t-\tau_{0}-\cdots-\tau_{N}} \mathrm{~d} \tau_{0} \mathrm{~d} \tau_{1} \cdots \mathrm{d} \tau_{N} \\
& \quad U\left(\tau_{0}+\tau_{1}+\cdots+\tau_{N}-t\right) R^{(N)}\left(A, \tau_{0}, \tau_{1}, \cdots, \tau_{N}\right) U\left(t-\tau_{0}-\tau_{1}-\cdots-\tau_{N}\right),
\end{aligned}
$$

where $A_{j} \in C_{b}^{0}\left(\mathbb{R}, \mathcal{A}_{m-j}\right)$ and $R^{(N)}\left(A, \tau_{1}, \cdots, \tau_{N}\right) \in C_{b}^{0}\left(\mathbb{R}^{N+1}, \mathcal{A}_{m-N-1}\right)$. Now we remark that the remainder term is as smoothing as we want by taking $N$ large enough, so the algebra being stable by smoothing perturbations we get Assumption II. 


\section{Proof of Lemma 2.16}

We reproduce here the proof given in the lecture notes by Giorgilli Gio (in particular the technical results are contained in Appendix A). A general presentation containing also the results that we use here can be found in Sie89.

We start by stating without proof a simple Lemma.

Lemma C.1. Let $\mathbf{e}_{1}, \ldots, \mathbf{e}_{d}$ and $\mathbf{e}_{1}^{\prime}, \ldots, \mathbf{e}_{d}^{\prime}$ be two basis of $\mathbb{Z}^{d}$; then the matrix $M=\left(M_{i j}\right)$ s.t. $\mathbf{e}_{i}^{\prime}=\sum_{j} M_{i j} \mathbf{e}_{j}$ is unimodular with integer entries.

Then one has the following corollary.

Corollary C.2. A collection of vectors $\mathbf{e}_{j} \in \mathbb{Z}^{d}, j=1, \ldots, d$, is a basis of $\mathbb{Z}^{d}$ if and only if the determinant of the matrix having $\mathbf{e}_{j}$ as rows is 1.

The corollary immediately follows from Lemma C.1 and the remark that such a property holds for the canonical basis of $\mathbb{Z}^{d}$.

Define now the resonance modulus $\mathcal{M}_{\nu}$ of $\nu$ by

$$
\mathcal{M}_{\nu}:=\left\{k \in \mathbb{Z}^{d}: \nu \cdot k=0\right\} .
$$

This is a discrete subgroup of $\mathbb{R}^{d}$ which satisfies

$$
\operatorname{span}\left(\mathcal{M}_{\nu}\right) \cap \mathbb{Z}^{d}=\mathcal{M}_{\nu} .
$$

Let $0 \leq r \leq d-1$ be the dimension of $\mathcal{M}_{\nu}$. It is well known that any discrete subgroup of $\mathbb{R}^{d}$ admits a basis. Let $\mathbf{e}_{1}, \ldots, \mathbf{e}_{r}$, be a basis of $\mathcal{M}_{\nu}$, and remark that the vectors $\mathbf{e}_{j}$ have integer components. Then the following result hold 3 .

Lemma C.3. There exist $\tilde{d}:=d-r$ vectors $\mathbf{u}_{1}, \ldots, \mathbf{u}_{\tilde{d}}$ with integer entries, such that $\mathbf{e}_{1}, \ldots, \mathbf{e}_{r}, \mathbf{u}_{1}, \ldots, \mathbf{u}_{\tilde{d}}$ form a basis of $\mathbb{Z}^{d}$.

Then one obtains immediately the following

Corollary C.4. Let $M$ be the matrix with rows given by the vectors $\mathbf{e}_{j}$ and the vectors $\mathbf{u}_{j}$; define $\check{\nu}:=M \nu$, then one has $\check{\nu}_{i}=0, \forall i=1, \ldots, r$, while $\tilde{\nu}_{i}:=\check{\nu}_{r+i}, i=1, \ldots, \tilde{d}$ are independent over the rationals.

Proof of Lemma 2.16. Consider the matrix $M^{-1}$ : since $M$ is unimodular with integer entries, the same is true for $M^{-1}$, and one has $\nu=M^{-1} \check{\nu}$; however, since the first $r$ components of $\check{\nu}$ vanish, such an expression reduces to a linear combination of vectors with integer entries, the coefficients of the combination being $\tilde{\nu}_{1}, \ldots, \tilde{\nu}_{\tilde{d}}$.

\footnotetext{
${ }^{3}$ this can be found as Theorem 31 in Sie89, or as Lemma A.6 in Gio
} 


\section{References}

[Arn89] V. Arnold. Mathematical methods of classical mechanics. Graduate Texts in Mathematics, 60. Springer-Verlag, New York, 1989.

[Bam17a] D. Bambusi. Reducibility of 1-d Schrödinger equation with time quasiperiodic unbounded perturbations, I. Trans. Amer. Math. Soc., 2017. doi:10.1090/tran/7135.

[Bam17b] D. Bambusi. Reducibility of 1-d Schrödinger equation with time quasiperiodic unbounded perturbations, II. Comm. Math. Phys., 353(1):353-378, 2017. doi:10.1007/s00220-016-2825-2.

[BBM14] P. Baldi, M. Berti, and R. Montalto. KAM for quasi-linear and fully nonlinear forced perturbations of Airy equation. Math. Ann., 359(1-2):471-536, 2014.

[BGMR17] D. Bambusi, B. Grébert, A. Maspero, and D. Robert. Reducibility of the quantum harmonic oscillator in $d$-dimensions with polynomial time dependent perturbation. February 2017. arXiv:1702.05274.

[BJ98] J. M. Barbaroux and A. Joye. Expectation values of observables in time-dependent quantum mechanics. J. of Statistical Physics, 90-5/6:1225-1249, 1998.

[Bou99] J. Bourgain. Growth of Sobolev norms in linear Schrödinger equations with quasi-periodic potential. Communications in Mathematical Physics, 204(1):207-247, 1999.

[BJLPN] L. Bunimovich, H. R. Jauslin, J. L. Lebowitz, A. Pellegrinotti and P. Nielaba. Diffusive energy growth in classical and quantum driven oscillators. J. Statist. Phys., 62(3-4):793-817, 1991.

[CdV79] Y. Colin de Verdière. Sur le spectre des opérateurs elliptiques à bicaractéristiques toutes périodiques. Comment. Math. Helv., 54(3):508-522, 1979.

[Del10] J.M. Delort. Growth of Sobolev norms of solutions of linear Schrödinger equations on some compact manifolds. Int. Math. Res. Notices, 12: 2305-2328, 2010.

[Del14] J.-M. Delort. Growth of Sobolev norms for solutions of time dependent Schrödinger operators with harmonic oscillator potential. Comm. Partial Differential Equations, 39(1):1-33, 2014. 
[DG97] J. Dereziński and C. Gérard. Scattering theory of classical and quantum $N$-particle systems. Texts and Monographs in Physics. Springer-Verlag, Berlin, 1997.

[DLS08] P. Duclos, O. Lev, and P. Šťovíček. On the energy growth of some periodically driven quantum systems with shrinking gaps in the spectrum. J. Stat. Phys., 130(1):169-193, 2008.

[EK09] H. L. Eliasson and S. B. Kuksin. On reducibility of Schrödinger equations with quasiperiodic in time potentials. Comm. Math. Phys., 286(1):125-135, 2009.

[FZ12] D. Fang and Q. Zhang. On growth of Sobolev norms in linear Schrödinger equations with time dependent Gevrey potential. J. Dynam. Differential Equations, 24(2):151-180, 2012.

[Gio] A. Giorgilli. Lecture notes on Hamiltonian systems and perturbations. URL: http://www.mat.unimi.it/users/antonio/hamsys/hamsys.html.

[GP16] B. Grébert and E. Paturel. On reducibility of quantum harmonic oscillator on $\mathbb{R}^{d}$ with quasiperiodic in time potential. ArXiv e-prints, March 2016. arXiv:1603.07455.

[Gui85] V. Guillemin. A new proof of Weyl's formula on the asymptotic distribution of eigenvalues. Advances in Math., 55:131-160, 1985 .

[Hör85] L. Hörmander. The analysis of linear partial differential operators I-III. Grundlehren der mathematischen Wissenschaften 256. Springer-Verlag, 1985.

[How89] J. Howland. Floquet operators with singular spectrum. I, II. Ann. Inst. H. Poincaré Phys. Théor. , 50(3): 309-323, 325334, 1989.

[How92] J. Howland. Stability of quantum oscillators. J. Physics, A25:5177-5181, 1992.

[HR82a] B. Helffer and D. Robert. Asymptotique des niveaux d'énergie pour des hamiltoniens à un degré de liberté. Duke Math. J., 49(4):853-868, 1982.

[HR82b] B. Helffer and D. Robert. Propriétés asymptotiques du spectre d'opérateurs pseudodifférentiels sur $\mathbf{R}^{n}$. Comm. Partial Differential Equations, 7(7):795-882, 1982. 
[IPT05] G. Iooss, P. I. Plotnikov, and J. F. Toland. Standing waves on an infinitely deep perfect fluid under gravity. Arch. Ration. Mech. Anal., 177(3):367-478, 2005.

[Joy94] A. Joye. Absence of absolutely continuous spectrum of Floquet operators. J. Stat. Phys., 75:929-952, 1994.

[Joy96] A. Joye. Upper bounds for the energy expectation in timedependent quantum mechanics. J. Stat. Phys., 85:575-606, 1996.

[Mon17] R. Montalto. On the growth of Sobolev norms for a class of linear Schrödinger equations on the torus with superlinear dispersion. Preprint, 2017.

[MR17] A. Maspero and D. Robert. On time dependent Schrödinger equations: Global well-posedness and growth of Sobolev norms. Journal of Functional Analysis, 273(2):721 - 781, 2017. doi:10.1016/j.jfa.2017.02.029

[Nen97] G. Nenciu. Adiabatic theory: stability of systems with increasing gaps. Annales de l'I. H. P, 67-4:411-424, 1997.

[PT01] P. I. Plotnikov and J. F. Toland. Nash-Moser theory for standing water waves. Arch. Ration. Mech. Anal., 159(1):1-83, 2001.

[Sie89] C. Siegel. Lectures on the geometry of numbers. SpringerVerlag, Berlin, 1989.

[Rob87] D. Robert. Autour de l'approximation semi-classique. PM 68. Birkhäuser, 1987.

[Tay91] M. Taylor. Pseudodifferential operators and nonlinear PDE. PM 100. Birkhäuser, 1991.

[Wan08] W.-M. Wang. Logarithmic bounds on Sobolev norms for time dependent linear Schrödinger equations. Communications in Partial Differential Equations, 33(12):2164-2179, 2008. 